\title{
Effect of High-Flow Nasal Cannula on Sleep- disordered Breathing and Sleep Quality in Patients With Acute Stroke
}

\author{
Nobuto Nakanishi ${ }^{1}$, Yasuhiro Suzuki ${ }^{2}$, Manabu Ishihara ${ }^{1}$, Yoshitoyo Ueno ${ }^{1}$, Natsuki Tane ${ }^{3}$, Yumiko \\ Tsunano $^{1}$, Taiga Itagaki ${ }^{1}$, Jun Oto ${ }^{1}$ \\ 1. Emergency and Critical Care Medicine, Tokushima University Hospital, Tokushima, JPN 2. Emergency and Critical \\ Care Center, Kurashiki Central Hospital, Kurashiki, JPN 3. Emergency and Disaster Medicine, Tokushima University \\ Hospital, Tokushima, JPN
}

Corresponding author: Nobuto Nakanishi,_nobuto_nakanishi@yahoo.co.jp

\section{Abstract \\ Introduction}

Sleep-disordered breathing (SDB) is common after stroke. Although the standard treatment of SDB is continuous positive airway pressure (CPAP) ventilation, the patient's intolerance and discomfort result in low adherence rates. Alternatively, high-flow nasal cannula (HFNC) may be useful as it reduces upper airway collapse with low level of positive pressure and well tolerability. The aim of this study was to investigate whether HFNC therapy reduces SDB and improves sleep quality with higher compliance rate.

\section{Methods}

We included acute stroke patients with SDB for the assessment of apnea-hypopnea index (AHI) $>5 / \mathrm{h}$ using WatchPAT 200 (Itamar Medical Ltd, Caesarea, Israel). Patients who met inclusion criteria received HFNC therapy (40 L/min) with monitoring by WatchPAT. AHI, oxygen desaturation index (ODI), sleep efficiency, and rapid eye movement (REM) sleep were compared in patients with and without HFNC therapy. We also evaluated the patient's comfort of HFNC therapy (discomfort or not).

\section{Results}

Among 17 patients assessed for AHI, 12 received HFNC therapy. HFNC therapy was not adhered in two patients due to intolerance. Eight patients remained for final analysis. There were no differences in SDB and sleep quality with and without HFNC therapy as follows: HFNC therapy vs control; AHI $24.9 \pm 20.1$ vs $21.3 \pm$ $15.0 / \mathrm{h}(\mathrm{p}=0.63)$, ODI $16.2 \pm 16.5$ vs $12.9 \pm 12.3 / \mathrm{h}(\mathrm{p}=0.54)$, sleep efficiency $80.4 \pm 12.9$ vs $87.1 \pm 6.2(\mathrm{p}=$ $0.28)$, percentage of REM sleep $19.4 \% \pm 9.6 \%$ vs $27.6 \% \pm 8.9 \%(p=0.07)$. Two patients $(17 \%)$ complained of discomfort among eight patients.

Received 06/24/2020 Review began 06/25/2020 Review ended 07/12/2020 Published 07/20/2020

\section{() Copyright 2020}

Nakanishi et al. This is an open access article distributed under the terms of the Creative Commons Attribution License CC-BY 4.0., which permits unrestricted use, distribution, and reproduction in any medium, provided the original author and source are credited.

\section{Conclusion}

HFNC therapy did not improve SDB and sleep quality. Nonadherence and discomfort were observed in HFNC therapy. We need a large trial to confirm this result.

\section{Categories: Internal Medicine, Neurology, Pulmonology}

Keywords: apnea-hypoxia index, high-flow nasal cannula, sleep-disordered breathing, stroke, adherence, oxygen desaturation index, sleep efficiency, rapid eye movement sleep

\section{Introduction}

Sleep-disordered breathing (SDB) is common after stroke, which is primarily characterized by upper airway obstruction (obstructive sleep apnea syndrome: OSA), and only 7\% of patients primarily having loss of central respiratory drive (central sleep apnea syndrome: central SAS) [1] .The prevalence of SDB after stroke is up to $72 \%$ compared to $33 \%$ in the general population $[1,2]$. SDB occurring after stroke has been shown to increase stroke-related mortality and morbidity [3]. The presence of SDB in stroke patients is associated with worse functional outcomes, longer duration of hospitalization, and more psychiatric and cognitive dysfunction [4].

The current standard of treatment for SDB is continuous positive airway pressure (CPAP) ventilation [5]. Emerging evidence suggests that CPAP ventilation therapy in poststroke patients leads to faster functional recovery and reduction in length of hospital stay and frequency of rehospitalization [6,7]. Moreover, it has been reasonably assumed that early respiratory support is more effective than late intervention in acute stroke patients. However, $25 \%-50 \%$ of patients with SDB will refuse or not tolerate the use of CPAP ventilation therapy due to complications and discomfort [8]. Alternatively, high-flow nasal cannula (HFNC) therapy may be useful as it can reduce upper airway collapse with low level of positive pressure [9]. 
Moreover, HFNC therapy washes out nasopharyngeal dead space and decreases the level of carbon dioxide [10]. Because of low pressure and no requirement of tight-fitting mask, HFNC therapy may achieve a higher compliance rate in SDB treatment.

We hypothesized that HFNC therapy can attenuate SDB and improve SDB and sleep quality in acute stroke patients with higher compliance rate. Moreover, no previous study has investigated the effect of HFNC therapy on SDB in acute stroke patients despite the associated morbidity and mortality [3]. In the present study, we investigated the effect of HFNC therapy on the severity of SDB and sleep characteristics in patients with acute stroke.

\section{Materials And Methods \\ Study design}

From May 2017 to February 2018, we conducted a pilot study at the Stroke Care Unit of Tokushima University Hospital. This study was approved by the clinical research ethics committee of Tokushima University Hospital (approval number: 2665) and registered as a clinical trial (UMIN-Clinical Trials Registry: 000040190). Written informed consent was obtained from patients or their authorized surrogate decisionmakers.

\section{Patient population}

During the study period, we screened patients with suspected SDB using nursing records at the Stroke Care Unit in Tokushima University Hospital. If patients were considered to have SDB, the screening test was conducted at nighttime for $8 \mathrm{~h}$ to evaluate the apnea-hypopnea index (AHI) using a wearable sleep monitor. Patients who met the criteria of AHI $>5 / \mathrm{h}$ were included in this study. We excluded patients based on the following exclusion criteria: age $<18$ years, SAS with suspected other conditions (primary neuromuscular disease, heart failure, and narcolepsy), past history of SAS, patients having unsuccessful recording in any WatchPAT channel, and the influence to WatchPAT, including catecholamine use, permanent pacemaker, and sustained nonsinus arrhythmia.

\section{High-flow nasal cannula}

On the next day of screening, HFNC therapy was performed at nighttime for $8 \mathrm{~h}$, which is the same duration used for the screening measurement. In HFNC therapy, we used the Optiflow ${ }^{\mathrm{TM}}$ system, which incorporated an $\mathrm{O}_{2}$ /air blender and a heated humidifier (MR850, Fisher \& Paykel Healthcare, Auckland, New Zealand). The MR850 was set in an invasive mode at a temperature of $40^{\circ} \mathrm{C}$. The flow was set at $40 \mathrm{~L} / \mathrm{min}$, and then the flow was titrated to $30 \mathrm{~L} / \mathrm{min}$ as the minimum flow to generate a pneumatic sprint effect according to the patient's acceptance. The fraction of inspired oxygen was set at the beginning to maintain an $\mathrm{SpO}_{2}$ level of $>93 \%$.

\section{WatchPAT}

To assess the severity of SDB and sleep quality, we used the WatchPAT (WatchPAT 200U, Itamar Medical Ltd, Caesarea, Israel) on two consecutive nights at the first screening and during HFNC therapy. The WatchPAT is a device worn around the wrist with a finger-mounted probe and a snoring sensor on the chest. The device has been approved by the Food and Drug Administration and used as a type 3 monitoring device by the American Academy of Sleep Medicine (AASM) [11]. An automatic algorithm analyzes the WatchPAT data using its original software (ZZZ PAT version 4.4.65.3, Itamar Medical Ltd, Caesarea, Israel). The WatchPAT can measure peripheral arterial tone (PAT), heart rate, oxygen saturation, body position, respiratory disturbance index (RDI), AHI, 4\% oxygen desaturation index (ODI), total sleep time, sleep efficiency, rapid eye movement (REM) sleep, and non-REM sleep. RDI is calculated from the number of respiratory events per hour, which meets any of the following three criteria: (1) reduction in PAT amplitude with increased pulse rate, (2) reduction in PAT amplitude with a 3\% oxygen desaturation, and (3) 4\% oxygen desaturation. AHI is calculated from RDI, and the severity is based on the AASM definition (mild: 5-15/h, moderate: $15-30 / \mathrm{h}$, and severe: $>30 / \mathrm{h})$. ODI is $4 \%$ desaturation events per hour.

\section{Outcomes}

As primary outcomes, we compared the AHI, ODI, sleep efficiency, and percentage of REM sleep with and without HFNC therapy. Secondary outcomes included other variables measured by WatchPAT and the patient's adherence and discomfort to HFNC therapy.

\section{Statistical analysis}

Continuous data are presented as mean \pm standard deviation or median [interquartile range (IQR)], as appropriate, whereas categorical data are presented as number (\%). Variables were compared using a paired t-test and the Wilcoxon signed-rank test. Sample size was not calculated a priori due to a pilot study [12]. Data analyses were conducted using JMP version 13.1.0 (SAS Institute Inc., Cary, NC). All statistical tests were two-tailed, and the chosen type 1 error rate was a $p$ value of $<0.05$. 


\section{Cureus}

\section{Results}

A total of 17 patients were assessed for SDB. Two patients did not meet the inclusion criteria of AHI $<5 / \mathrm{h}$. Measurement error was observed in two patients. In one patient, the WatchPAT was dislodged unintentionally during sleep. In total, 12 patients were treated by HFNC at $40 \mathrm{~L} / \mathrm{min}$, and one patient was titrated to $30 \mathrm{~L} / \mathrm{min}$ according to the patient's acceptance. Among the twelve patients, eight were included for final analysis because two recordings resulted in insufficient data and HFNC therapy was not adhered in two patients due to intolerance (Figure 1).

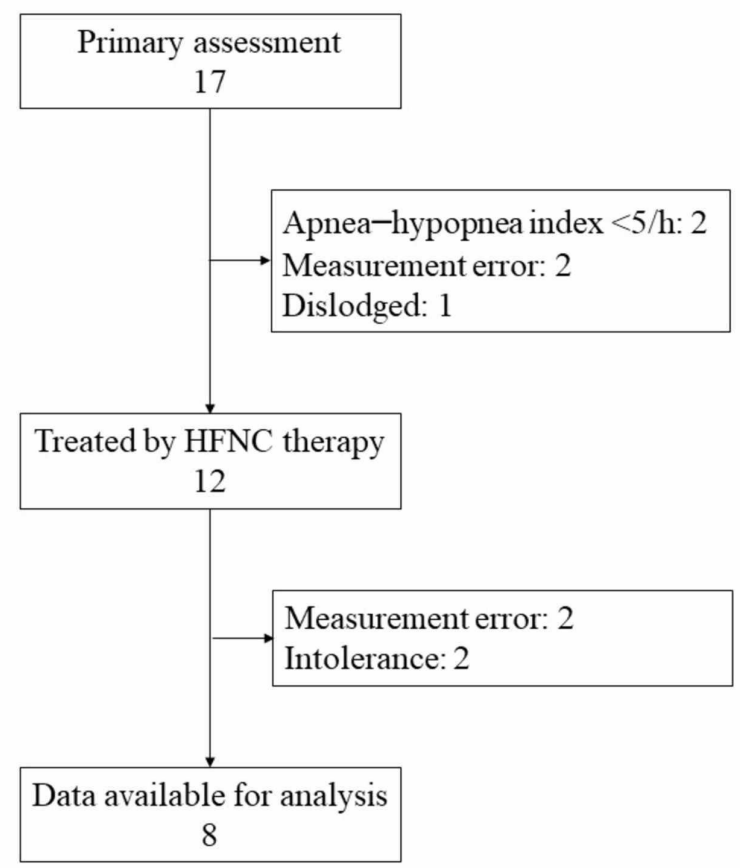

FIGURE 1: Flowchart of patients included in this study.

A total of 17 patients were assessed for sleep apnea syndrome, and 12 patients were included for HFNC therapy. Eight patients remained for final analysis.

HFNC: high-flow nasal cannula.

Table 1 summarizes the characteristics of the study patients. 


\section{Cureus}

\begin{tabular}{|c|c|c|c|c|c|c|c|c|c|c|}
\hline Patients & A & B & c & D & $\mathrm{E}$ & $\mathrm{F}$ & G & H & Mean & SD \\
\hline \multicolumn{11}{|l|}{ Characteristics } \\
\hline Age, year & 79 & 65 & 90 & 57 & 73 & 62 & 50 & 70 & 68 & 12 \\
\hline Gender & M & M & $\mathrm{F}$ & M & M & M & M & M & & \\
\hline BMl, $\mathrm{kg} / \mathrm{m}^{2}$ & 21.7 & 28.6 & 23.6 & 21.0 & 21.5 & 32.2 & 31.4 & 20.5 & 25.0 & 4.6 \\
\hline Gcs & 15 & 15 & 14 & 15 & 14 & 6 & 15 & 15 & 14 & 3 \\
\hline NHISS & 10 & 0 & 24 & 8 & 16 & 23 & 1 & 8 & 11 & 8 \\
\hline \multicolumn{11}{|c|}{ Sleep disorders } \\
\hline AHl, evens/h & 7.4 & 7.3 & 8.0 & 20.6 & 17.6 & 24.7 & 35.2 & 49.3 & 21.3 & 14.0 \\
\hline Severity & Mild & Mild & Mild & Moderate & Moderate & Moderate & Severe & Severe & & \\
\hline
\end{tabular}

\section{TABLE 1: Patient characteristics}

BMI: body mass index, GCS: Glasgow Coma Scale, NIHSS: National Institute of Health Stroke Scale, AHI: apnea-hypopnea index, SD: standard deviation.

The mean age of the patients was $68 \pm 12$ years, and seven patients were males. Six patients had ischemic stroke, and one patient had intracerebral hemorrhage (patient E) or subarachnoid hemorrhage (patient B). Only one patient required surgery before the measurement (patient $\mathrm{B}$ ). The measurement was conducted on day 3 (IQR: 2-4) after the admission to the Stroke Care Unit. Two patients complained of discomfort after the HFNC therapy (patients E and G). During the measurements, the body position was not different between HFNC therapy vs control (spine position: $88 \%$ vs $78 \%, \mathrm{p}=0.39$ ).

There were no differences between patients with and without HFNC therapy in primary outcomes (Figure 2, Table 2).
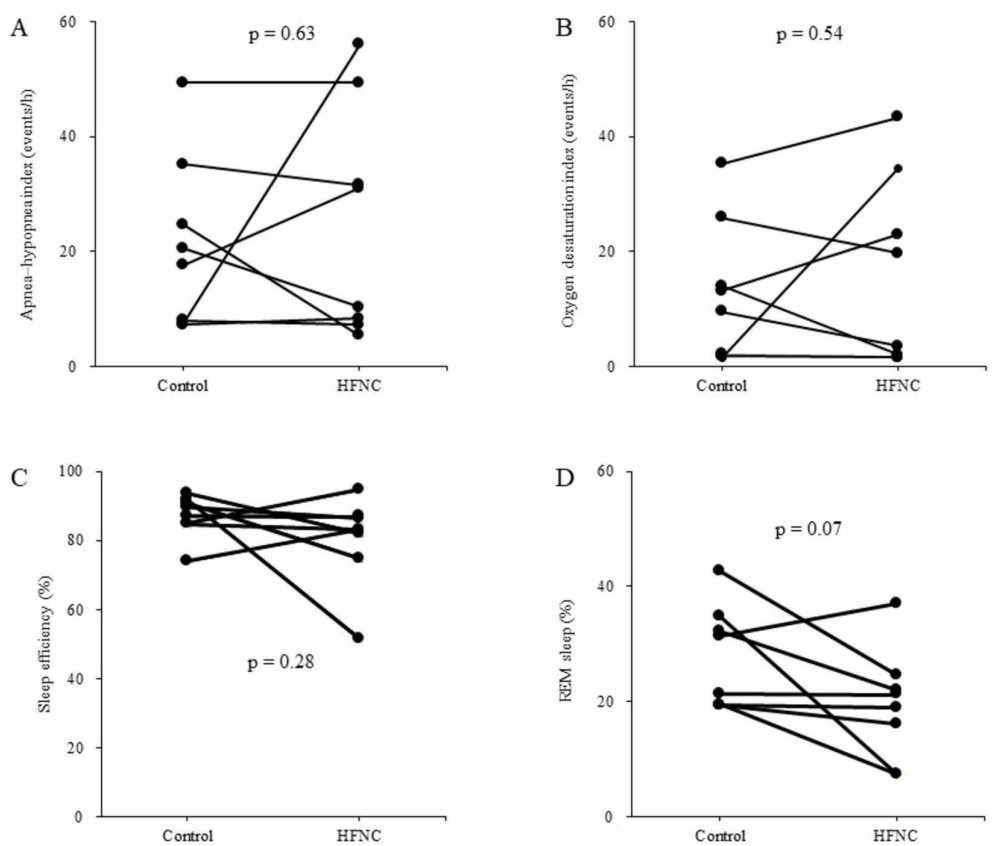

FIGURE 2: Results of actigraphy before and after HFNC therapy.

A, Apnea-hypopnea index. B, Oxygen desaturation index. C, Sleep efficiency. D, REM sleep. No differences were observed between patients with and without HFNC. Data are compared using the Wilcoxon signed-rank test. 


\begin{tabular}{|c|c|c|c|c|c|c|c|c|}
\hline \multirow[b]{2}{*}{ Patients } & \multicolumn{2}{|c|}{ AHI (events/h) } & \multicolumn{2}{|c|}{ ODI (events/h) } & \multicolumn{2}{|c|}{ Sleep efficiency $(\%)$} & \multicolumn{2}{|c|}{ REM sleep (\%) } \\
\hline & Control & HFNC & Control & HFNC & Control & HFNC & Control & HFNC \\
\hline A & 7.4 & 8.3 & 2.2 & 1.6 & 74.0 & 83.3 & 19.4 & 16.1 \\
\hline B & 7.3 & 56.0 & 1.5 & 34.5 & 91.8 & 51.7 & 19.6 & 7.4 \\
\hline c & 8.0 & 7.2 & 1.8 & 1.6 & 93.7 & 82.2 & 19.3 & 19.0 \\
\hline D & 20.6 & 10.3 & 9.5 & 3.5 & 90.9 & 74.9 & 32.3 & 22.0 \\
\hline $\mathrm{E}$ & 17.6 & 31.0 & 13.1 & 23.0 & 84.8 & 83.1 & 31.3 & 37.1 \\
\hline $\mathrm{F}$ & 24.7 & 5.4 & 14.0 & 2.0 & 89.6 & 86.5 & 34.8 & 7.4 \\
\hline G & 35.2 & 31.6 & 25.9 & 19.7 & 87.0 & 87.0 & 42.8 & 24.6 \\
\hline $\mathrm{H}$ & 49.3 & 49.3 & 35.3 & 43.3 & 85.1 & 94.6 & 21.4 & 21.3 \\
\hline Mean & 21.3 & 24.9 & 12.9 & 16.2 & 87.1 & 80.4 & 27.6 & 19.4 \\
\hline SD & 15.0 & 20.1 & 12.3 & 16.5 & 6.2 & 12.9 & 8.9 & 9.6 \\
\hline
\end{tabular}

\section{TABLE 2: Results of actigraphy}

AHI: apnea-hypopnea index, ODI: oxygen desaturation index, REM: rapid eye movement, HFNC: high-flow nasal cannula, SD: standard deviation.

Regarding other variables, the results for HFNC therapy vs control were as follows: total sleep time $451 \pm 75$ vs $497 \pm 80 \mathrm{~min}(\mathrm{p}=0.27)$, percentage of non-REM sleep $79.2 \% \pm 8.3 \%$ vs $72.4 \% \pm 8.9 \%(\mathrm{p}=0.06)$, and RDI $25.7 \pm 19.6$ vs $21.8 \pm 14.5 / \mathrm{h}(\mathrm{p}=0.60)$. There was no difference in respiratory and circulatory parameters between patients with and without HFNC therapy as follows: $\mathrm{SpO}_{2} 94.8 \% \pm 1.3 \%$ vs $95.1 \% \pm 1.1 \%(\mathrm{p}=0.53)$ and heart rate $68 \pm 14$ vs $68 \pm 14 / \mathrm{min}(\mathrm{p}=0.98)$.

\section{Discussion}

In the present study, we observed that HFNC therapy did not improve SDB and sleep quality in patients with acute stroke. To our knowledge, this is the first study to investigate the effect of HFNC therapy on SDB and sleep quality in patients with acute stroke. Although HFNC therapy is commonly more acceptable than CPAP ventilation therapy, four (34\%) of twelve patients with HFNC therapy complained of discomfort and two patients could not continue to receive HFNC therapy, indicating insufficient adherence to the respiratory therapy.

The end-expiratory pressure generated by HFNC therapy may not be sufficient to prevent the collapse of upper airway. Although we did not measure the end-expiratory pressure, the pharyngeal pressure generated by the HFNC flow of $40 \mathrm{~L} / \mathrm{min}$ is around $1.5 \mathrm{~cm} \mathrm{H}_{2} \mathrm{O}$ [13]. In general, $5-15 \mathrm{~cm} \mathrm{H}_{2} \mathrm{O}$ is required to stabilize the symptom of OSA during CPAP ventilation therapy $[14,15]$. It is understandable that the pressure generated by HFNC therapy was not effective in reducing upper airway collapse. However, McGinley et al. reported that a nasal insufflation of $20 \mathrm{~L} / \mathrm{min}$ improved upper airway patency [9]. The difference may be attributed to the study population. In our study, we investigated the effect of HFNC therapy in patients with acute stroke. Therefore, central SAS, or at least the complex of central and obstructive, may be included in the subjects. The use of HFNC therapy may negatively affect those patients having central SAS. HFNC therapy washes out nasopharyngeal dead space and consequently reduces carbon dioxide levels. This condition may lead to a poor response from the central nerve and may aggravate the central SAS. Another study also reported a different result of a device between stroke patients and general population. That study used nasal expiratory positive airway pressure, which is a valve inserted into each nostril. The device did not reduce $\mathrm{AHI}$ in stroke patients, but it was useful in the general population [16,17].

HFNC therapy did not change the sleep efficiency and the percentage of REM sleep. In stroke patients, sleep quality is often impaired [18]. In a meta-analysis, sleep efficiency was found to be lower in stroke patients than in controls (69\%-79\% vs 78\%-78\%, p = 0.006) [19]. Improving sleep quality is essential in stroke patients because poor sleep quality is associated with impaired functional status [20]. However, HFNC therapy was insufficient in improving sleep quality possibly due to unchanged AHI and noise-induced sleep disruption. Although we did not measure the noise level, the noise level generated by HFNC therapy is 50-70 
$\mathrm{dB}$ at $40 \mathrm{~L} / \mathrm{min}$ and exceeds the recommended level of $\angle 30 \mathrm{~dB}$ at night [21,22]. Noise-reducing strategies are required to facilitate sleep promotion during HFNC therapy in the acute care unit [23].

The important finding of this study was not the high acceptance ratio of HFNC therapy. In CPAP ventilation therapy, a low adherence rate of $20 \%-40 \%$ is a problem, and the use of HFNC therapy is expected to have a better compliance rate [8]. However, two patients did not tolerate HFNC therapy, with two more complaining of the discomfort. The discomfort is attributed to the nasal irritation due to the high amount of flow and temperature. In a previous study using $20 \mathrm{~L} / \mathrm{min}$ of flow through an open nasal cannula, all patients tolerated the flow. The flow of $40 \mathrm{~L} / \mathrm{min}$ may exceed the patient's tolerance. In fact, the flow of 30-60 L/min reportedly caused discomfort, especially in the population with less severe acute hypoxemic respiratory failure [24]. In our study, most of the patients had normal lung function, which may be related to the discomfort despite the severity of SDB.

There are several limitations in our study. First, the sample size was small. Second, we did not use polysomnography, which is a gold standard but expensive and difficult to conduct immediately outside of a specialized center, stressful in the head attachment, and difficult to attach on the head after head surgery [25]. In our study, polysomnography was not suited in the early phase of stroke care. In most of the previous studies, WatchPAT correlated with polysomnography (correlation coefficient: $0.87-0.94 ; \mathrm{p}<0.01$ ), and the sensitivity/specificity values were $100 \% / 75 \%$ and $80 / 100 \%$ at an AHI of 5 and 30/h, respectively [26]. Therefore, our data are still reliable. Third, we included stroke patients who may have atherosclerosis in peripheral blood vessels. WatchPAT may underestimate sleep events in these subjects [27].

\section{Conclusions}

We evaluated the effect of HFNC therapy on SDB and sleep quality in patients with acute stroke. HFNC therapy did not change SDB and sleep quality, and nonadherence and discomfort were observed during the therapy. We need a large trial to confirm this result.

\section{Additional Information \\ Disclosures}

Human subjects: Consent was obtained by all participants in this study. The Clinical Research Ethics Committee of Tokushima University Hospital issued approval 2665. This study was approved by the Clinical Research Ethics Committee of Tokushima University Hospital (approval no 2665). Animal subjects: All authors have confirmed that this study did not involve animal subjects or tissue. Conflicts of interest: In compliance with the ICMJE uniform disclosure form, all authors declare the following: Payment/services info: All authors have declared that no financial support was received from any organization for the submitted work. Financial relationships: All authors have declared that they have no financial relationships at present or within the previous three years with any organizations that might have an interest in the submitted work. Other relationships: All authors have declared that there are no other relationships or activities that could appear to have influenced the submitted work.

\section{Acknowledgements}

The authors thank medical staff at stroke care unit of Tokushima University Hospital for their support.

\section{References}

1. Johnson KG, Johnson DC: Frequency of sleep apnea in stroke and TIA patients: a meta-analysis . J Clin Sleep Med. 2010, 6:131-137. 10.5664/jcsm.27760

2. Peppard PE, Young T, Barnet JH, Palta M, Hagen EW, Hla KM: Increased prevalence of sleep-disordered breathing in adults. Am J Epidemiol. 2013, 177:1006-1014. 10.1093/aje/kws342

3. Xie W, Zheng F, Song X: Obstructive sleep apnea and serious adverse outcomes in patients with cardiovascular or cerebrovascular disease: a PRISMA-compliant systematic review and meta-analysis. Medicine. 2014, 93:e336. 10.1097/md.0000000000000336

4. McKee Z, Auckley DH: A sleeping beast: obstructive sleep apnea and stroke. Cleve Clin J Med. 2019, 86:407415. 10.3949/ccjm.86a.18033

5. Mello-Fujita L, Kim LJ, Palombini Lde O, Rizzi C, Tufik S, Andersen ML, Coelho FM: Treatment of obstructive sleep apnea syndrome associated with stroke. Sleep Med. 2015, 16:691-696. 10.1016/j.sleep.2014.12.017

6. Martínez-García MA, Soler-Cataluña JJ, Ejarque-Martínez L, et al.: Continuous positive airway pressure treatment reduces mortality in patients with ischemic stroke and obstructive sleep apnea: a 5-year followup study. Am J Respir Crit Care Med. 2009, 180:36-41. 10.1164/rccm.200808-13410C

7. Kim H, Im S, Park JI, Kim Y, Sohn MK, Jee S: Improvement of cognitive function after continuous positive airway pressure treatment for subacute stroke patients with obstructive sleep apnea: a randomized controlled trial. Brain Sci. 2019, 9:252. 10.3390/brainsci9100252

8. Rotenberg BW, Murariu D, Pang KP: Trends in CPAP adherence over twenty years of data collection: a flattened curve. J Otolaryngol Head Neck Surg. 2016, 45:43. 10.1186/s40463-016-0156-0

9. McGinley BM, Patil SP, Kirkness JP, Smith PL, Schwartz AR, Schneider H: A nasal cannula can be used to treat obstructive sleep apnea. Am J Respir Crit Care Med. 2007, 176:194-200. 10.1164/rccm.200609-13360C 
10. Nishimura M: High-flow nasal cannula oxygen therapy in adults: physiological benefits, indication, clinical benefits, and adverse effects. Respir Care. 2016, 61:529-541. 10.4187/respcare.04577

11. Choi JH, Kim EJ, Kim YS, et al.: Validation study of portable device for the diagnosis of obstructive sleep apnea according to the new AASM scoring criteria: Watch-PAT 100. Acta Otolaryngol. 2010, 130:838-843. 10.3109/00016480903431139

12. Lancaster GA, Thabane L: Guidelines for reporting non-randomised pilot and feasibility studies . Pilot Feasibility Stud. 2019, 5:114. 10.1186/s40814-019-0499-1

13. Ritchie JE, Williams AB, Gerard C, Hockey H: Evaluation of a humidified nasal high-flow oxygen system, using oxygraphy, capnography and measurement of upper airway pressures. Anaesth Intensive Care. 2011, 39:1103-1110. 10.1177/0310057x1103900620

14. Andrade RG, Madeiro F, Piccin VS, et al.: Impact of acute changes in CPAP flow route in sleep apnea treatment. Chest. 2016, 150:1194-1201. 10.1016/j.chest.2016.04.017

15. Minnerup J, Ritter MA, Wersching H, et al.: Continuous positive airway pressure ventilation for acute ischemic stroke: a randomized feasibility study. Stroke. 2012, 43:1137-1139. 10.1161/strokeaha.111.637611

16. Wheeler NC, Wing JJ, O'Brien LM, et al.: Expiratory positive airway pressure for sleep apnea after stroke: a randomized, crossover trial. J Clin Sleep Med. 2016, 12:1233-1238. 10.5664/jcsm.6120

17. Berry RB, Kryger MH, Massie CA: A novel nasal expiratory positive airway pressure (EPAP) device for the treatment of obstructive sleep apnea: a randomized controlled trial. Sleep. 2011, 34:479-485. 10.1093/sleep/34.4.479

18. Da Rocha PC, Barroso MT, Dantas AA, Melo LP, Campos TF: Predictive factors of subjective sleep quality and insomnia complaint in patients with stroke: implications for clinical practice. An Acad Bras Cienc. 2013, 85:1197-1206. 10.1590/s0001-37652013005000053

19. Baglioni C, Nissen C, Schweinoch A, et al.: Polysomnographic characteristics of sleep in stroke: a systematic review and meta-analysis. PLoS One. 2016, 11:e0148496. 10.1371/journal.pone.0148496

20. Sonmez I, Karasel S: Poor sleep quality I related to impaired functional status following stroke . J Stroke Cerebrovasc Dis. 2019, 28:104349. 10.1016/j.jstrokecerebrovasdis.2019.104349

21. Kubo T, Nakajima H, Shimoda R, Seo T, Kanno Y, Kondo T, Tamai S: Noise exposure from high-flow nasal cannula oxygen therapy: a bench study on noise reduction. Respir Care. 2018, 63:267-273. 10.4187/respcare.05668

22. Itagaki T, Nishimura M: High-flow nasal cannula devices: noisier than we realize . Respir Care. 2018, 63:371372. $10.4187 /$ respcare.06155

23. Simons KS, Verweij E, Lemmens PMC, et al.: Noise in the intensive care unit and its influence on sleep quality: a multicenter observational study in Dutch intensive care units. Crit Care. 2018, 22:250. 10.1186/s13054-018-2182-y

24. Mauri T, Galazzi A, Binda F, et al.: Impact of flow and temperature on patient comfort during respiratory support by high-flow nasal cannula. Crit Care. 2018, 22:120. 10.1186/s13054-018-2039-4

25. Rundo JV: Obstructive sleep apnea basics. Cleve Clin J Med. 2019, 86:2-9. 10.3949/ccjm.86.s1.02

26. Zhang P, Zhang R, Zhao F, et al.: The prevalence and characteristics of obstructive sleep apnea in hospitalized patients with type 2 diabetes in China. J Sleep Res. 2016, 25:39-46. 10.1111/jsr.12334

27. Kinoshita T, Yahaba M, Terada J, et al.: Impact of arterial stiffness on WatchPAT variables in patients with obstructive sleep apnea. J Clin Sleep Med. 2018, 14:319-325. 10.5664/jcsm.6970 